\title{
Design principles for HgTe based Topological Insulator Devices
}

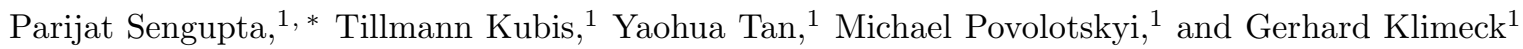 \\ ${ }^{1}$ Dept of Electrical and Computer Engineering, Purdue University, West Lafayette, IN, 47907
}

\begin{abstract}
The topological insulator properties of $\mathrm{CdTe} / \mathrm{HgTe} / \mathrm{CdTe}$ quantum wells are theoretically studied. The $\mathrm{CdTe} / \mathrm{HgTe} / \mathrm{CdTe}$ quantum well behaves as a topological insulator beyond a critical well width dimension. It is shown that if the barrier $(\mathrm{CdTe})$ and well-region $(\mathrm{HgTe})$ are altered by replacing them with the alloy $\mathrm{Cd}_{x} \mathrm{Hg}_{1-x} \mathrm{Te}$ of various stoichiometries, the critical width can be changed.The critical quantum well width is shown to depend on temperature, applied stress, growth directions and external electric fields. Based on these results, a novel device concept is proposed that allows to switch between a normal semiconducting and topological insulator state through application of moderate external electric fields.
\end{abstract}

PACS numbers:

Keywords:

\section{Introduction}

An insulator is conventionally defined as a material that does not conduct electricity. In most insulators the lack of electric conduction is explained using its bandstructure properties. The band theory predicts that an insulator has an energy gap separating the conduction and valence bands. As a result of this finite band-gap there are no electronic states to support the flow of current. Recently, materials that have an energy gap in bulk but possess gapless states bound to the sample surface or edge have been theoretically predicted and experimentally observed. [1] These states, in a time reversal invariant system are protected against perturbation and nonmagnetic disorder. 2 [5] Materials that support such states are known as topological insulators (TI). Examples of materials with such properties include $\mathrm{Bi}_{2} \mathrm{Te}_{3}, \mathrm{Bi}_{2} \mathrm{Se}_{3}$, $\mathrm{Bi}_{x} \mathrm{Sb}_{1-x}$ alloys, and $\mathrm{CdTe} / \mathrm{HgTe} / \mathrm{CdTe}$ quantum wells. $\mathrm{Bi}_{2} \mathrm{Te}_{3}, \mathrm{Bi}_{2} \mathrm{Se}_{3}$, and $\mathrm{Bi}_{x} \mathrm{Sb}_{1-x}$ belong to the class of $3-$ $\mathrm{D}$ topological insulators (3D-TI) and host bound states on their surface. 6 -8] CdTe-HgTe-CdTe quantum wells, which were the first predicted TIs are 2-D topological insulators (2-D TI). Unlike their 3D counterpart, they possess bound states at the edge of the quantum well. 90 [1] These conducting surface and edge states develop at the boundary between two insulators, where one is normal (NI) and the other of inverted band ordering. The surface states, which are subject to the details of the band properties of each involved material can mutually influence each other. It is therefore essential to theoretically study the surface states under various conditions.

This work proposes ways that can efficiently invert the band profile of a $\mathrm{CdTe} / \mathrm{HgTe} / \mathrm{CdTe}$ heterostructure and create bound edge states. Specifically, the transition from an NI to a TI through external adiabatic parameters, adjustable lattice constants, or modulation of the electronhole band coupling is the underlying theme. The paper is organized as follows: In Section III details of the $\mathrm{CdTe} / \mathrm{HgTe} / \mathrm{CdTe}$ structure and method to compute the

*Electronic address: psengupta@purdue.edu energy dispersion are described. Section[II] discusses various inverted band structures, device and material conditions that can lead to topologically protected conducting surface states. The concept of creating a switch from a topological insulator is developed here. The key results discussed in the paper are summarized in Section IV

\section{Materials and Methods}

An HgTe quantum well flanked by CdTe barriers has been shown to have edge states with topological insulator properties. 12 TI behaviour is possible because CdTe is a normal insulator and is placed in contact with an inverted insulator $\mathrm{HgTe}$. A representative sketch of the device is shown in Fig,1. CdTe is a wide band gap semiconductor $\left(E_{g}=1.606 \mathrm{eV}\right)$ with positive energy gap (NI) and a small lattice mismatch of $0.5 \%$ with $\mathrm{HgTe}$. CdTe, because of similar lattice constants is chosen as the barrier for the HgTe well region though in principle any normal ordered material would suffice. The normal valence and conduction band are reversed in their energetic order in $\mathrm{HgTe}$ as indicated in Fig. 1 and explained in the next paragraph.

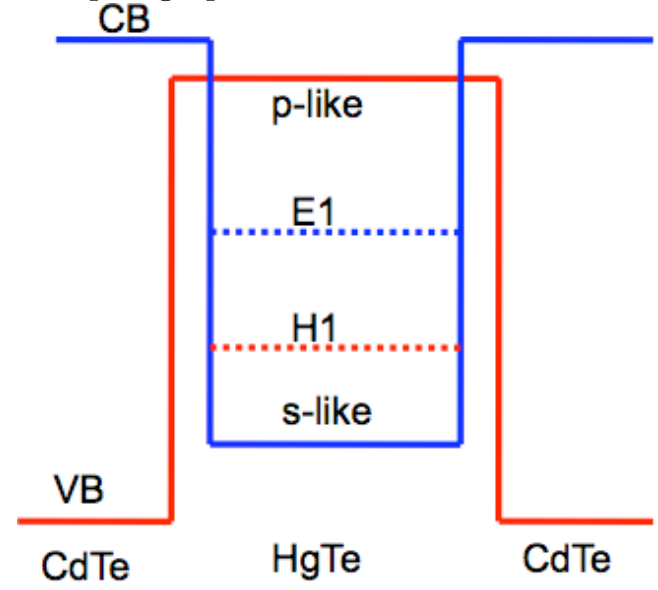

FIG. 1: Sketch of a CdTe/HgTe/CdTe quantum well heterostructure. The lowest conduction band (CB) state is labeled with $\mathrm{E} 1$ and the highest valence band (VB) state with H1.

The inversion of bands for the $\mathrm{CdTe} / \mathrm{HgTe} / \mathrm{CdTe}$ het- 
erostructure is achieved through the HgTe component. Both CdTe and HgTe belong to the zinc blende (ZB) structure with $\mathrm{T}_{d}$ point group symmetry. The highest valence and lowest conduction band is made up of $p$ and $s$ orbitals respectively. A normal band order at $\Gamma$ has lowest conduction band $(j=1 / 2)$ with $\Gamma_{6}$ symmetry above the top of the valence bands $(j=3 / 2)$ with $\Gamma_{8}$ symmetry. The $\Gamma_{6}$ state has $s$-type symmetry and the $\Gamma_{8}$ state has $p$-type symmetry. In a normal ordered material $\Gamma_{6}$ state is energetically higher than the $\Gamma_{8}$ state. This order is reversed in bulk $\mathrm{HgTe}$ at the $\Gamma$ point due to the high spin-orbit coupling and a significant Darwin term contribution. 13] The strong spin orbit coupling pushes the valence bands upwards while the Darwin term shifts the $s$-type conduction band down. The Darwin term can only influence the $s$-type bands. 14] The combined effect of spin orbit coupling and Darwin term yields an inverted band order at the $\Gamma$ point which flips the order of the high-symmetry $\Gamma_{6}$ and $\Gamma_{8}$ points for HgTe. [15] The energy gap at $\Gamma$ which is defined as

$$
E_{g}=E\left(\Gamma_{6}\right)-E\left(\Gamma_{8}\right)
$$

therefore turns out to be negative for HgTe. The normal and inverted band structures of CdTe and HgTe are illustrated in Fig. 2(a) and Fig. 2(b) respectively.

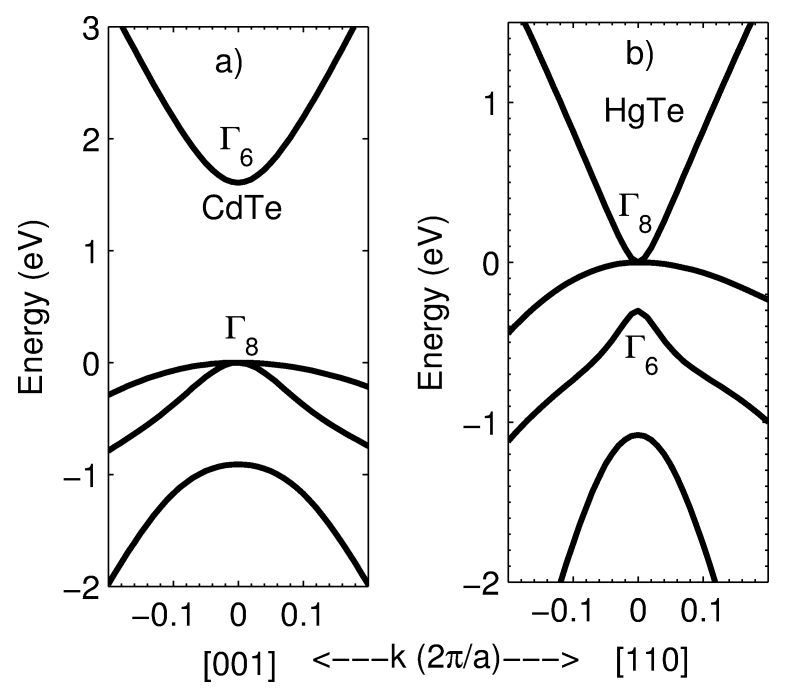

FIG. 2: Bulk band structure of CdTe (a) and $\operatorname{HgTe}(\mathrm{b})$. The ordering of the conduction and valence bands near the band gap at the $\Gamma$ point in $\mathrm{HgTe}$ (Fig. 2b) is opposite to the one in CdTe (Fig. 2a). In $\mathrm{HgTe}$, the hole state $\Gamma_{8}$ is above the electron state $\Gamma_{6}$.

In this work, electronic properties of the $\langle 001\rangle$ $\mathrm{CdTe} / \mathrm{HgTe} / \mathrm{CdTe}$ heterostructure are calculated within an 8-band k.p framework that includes a linear coupling between conduction and valence bands. [16, 17] In the calculations presented, the $z$-axis is normal to the heterostructure and is also the confinement direction. The valence band edge $E_{v}$, Luttinger parameters, and other related material properties are collected in Table [18]
The boundary conditions are imposed by setting the wave function to zero at the edge of the device. Strain is added to the electronic Hamiltonian using deformation potentials defined in the Bir-Pikus method. [19, 20]

TABLE I: 8-band k.p parameters for CdTe and HgTe. $E_{v}$, $E_{g}, P_{c v}$, and $\mathrm{V}_{s o}$ are in units of $\mathrm{eV}$. The remaining Luttinger parameters are dimensionless constants and the effective mass is in units of the free electron mass.

\begin{tabular}{|c|c|c|c|c|c|c|c|c|}
\hline Material & $E_{v}$ & $\gamma_{1}$ & $\gamma_{2}$ & $\gamma_{3}$ & $m^{*}$ & $E_{g}$ & $P_{c v}$ & $\mathrm{~V}_{s o}$ \\
\hline $\mathrm{CdTe}$ & -0.27 & 5.372 & 1.671 & 1.981 & 0.11 & 1.606 & 18.8 & 0.91 \\
\hline $\mathrm{HgTe}$ & 0.0 & -16.08 & -10.6 & -8.8 & -0.031 & -0.303 & 18.8 & 1.08 \\
\hline
\end{tabular}

\section{Results and Discussion}

Comparison with experiment: band gap and critical widthExperiments report that a $\mathrm{CdTe} / \mathrm{HgTe} / \mathrm{CdTe}$ quantum well heterostructure with a well width under $6.3 \mathrm{~nm}$ exhibits a normal band order with positive $E_{g} .[21,22]$. The calculation of the present work confirms that the conduction states at $\Gamma$ are indeed located above the valence states and the energy gap is positive (Fig. 4(a)). All band structure parameters used to reproduce the experimental observation were valid at $0 \mathrm{~K}$. When the well width is exactly $6.3 \mathrm{~nm}$, a Dirac system [23] is formed in the volume of the device (Fig. 31).

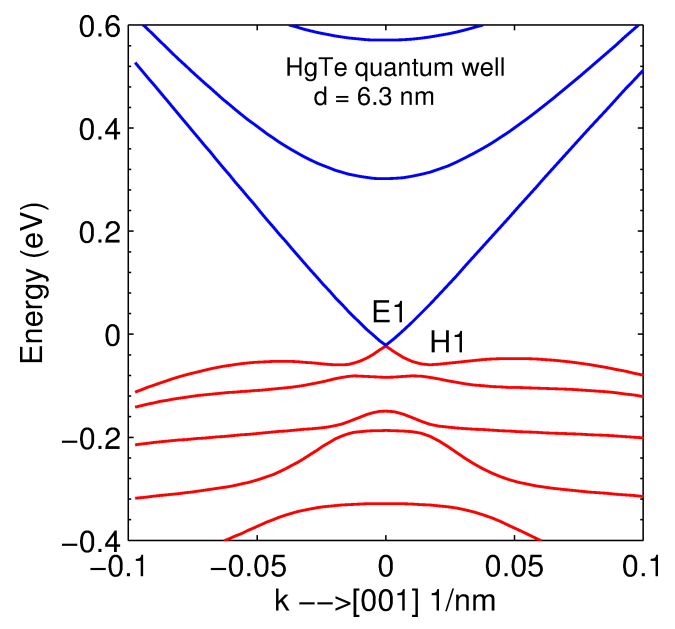

FIG. 3: Band structure of HgTe quantum well of thickness $6.3 \mathrm{~nm}$. At this width, the lowest conduction band (E1) and highest valence band (H1) at the $\Gamma$ point are equal.

Beyond this critical well width of $6.3 \mathrm{~nm}$, the heterostructure has its bands fully inverted. The band profile has a reverse ordering of the $s$-type and $p$-type orbitals (Fig. 4(c)) and $E_{g}<0$.

Accordingly, a nano-ribbon of width $100.0 \mathrm{~nm}$ formed by quantizing the quantum well in its in-plane direction has a positive band gap (as shown in Fig. 4b). Similarly, a nanoribbon of width $100.0 \mathrm{~nm}$ constructed out of an inverted quantum well possesses gap-less TI edge states. The band structure of this situation is illustrated in Fig. 4(d). 

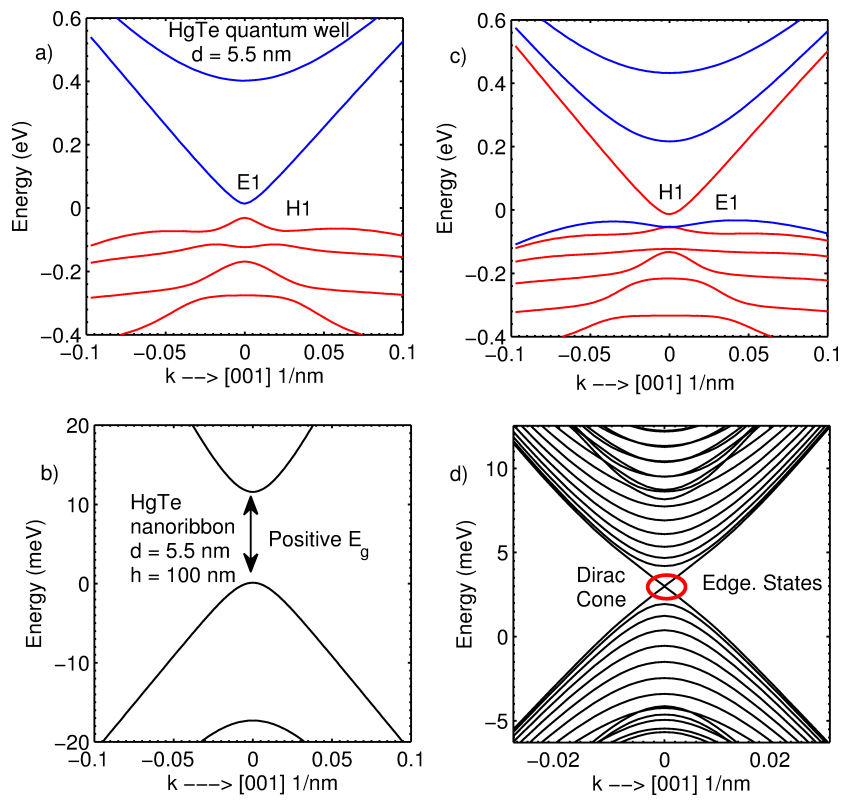

FIG. 4: Bandstructure of a HgTe quantum well of thickness $5.5 \mathrm{~nm}(\mathrm{a})$. A HgTe nano-ribbon formed out of this quantum well of thickness $5.5 \mathrm{~nm}$ and height of $100 \mathrm{~nm}$ shows a positive band gap. Fig. 4 shows the bandstructure of an inverted quantum well of thickness $10.0 \mathrm{~nm}$. The corresponding quantum wire has a linearly dispersing (Dirac-cone) edge states (d).

The corresponding absolute value of the squared edgestate wave functions is plotted in Fig. 5. The absolute value of the wave functions for the two edge states is maximum at the edge and gradually decay in to the bulk. This establishes that they belong exclusively to the edge states. In conclusion, the band-gap closing Dirac cone shown in Fig. 3 marks the transition from a positive band-gap to a negative one.

Band nature at finite momenta: The inversion of bands in the volume of the well is necessary for edge states with topological insulator behavior. It is important to note however, that the process of inversion happens only at the $\Gamma$ point. In the inverted dispersion plot (Fig. 4c), for momenta different from the $\Gamma$ point, the band labeled with "H1" progresses from $p$ to $s$-type. Similarly the band labeled with "E1" changes character from $s$ to $p$. Both the bands, at a finite momentum acquire atomic orbital characteristics associated with a normally ordered set of bands. TI behavior is therefore restricted to a special set of momentum points where the band structure is inverted. These set of points are collectively called the time-reversal-invariant-momentum (TRIM) points. [24]

Well thickness continuously tunes the TI properties: With increasing well width, the band gap decreases continuously until the HgTe well thickness reaches $6.3 \mathrm{~nm}$ (see Fig. 6). This is due to the diminishing confinement of the well's $s$ and $p$-type bands. For well thicker than $6.3 \mathrm{~nm}$, the confinement is small enough such

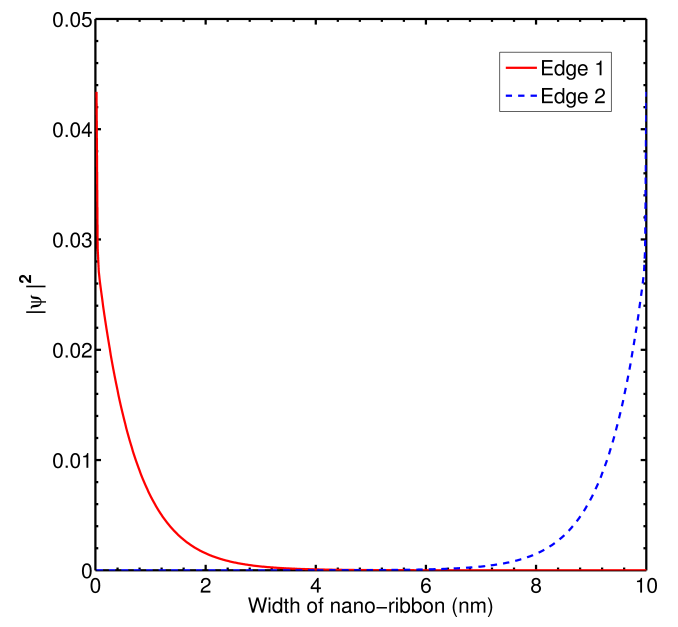

FIG. 5: Absolute value of the wave functions $|\psi|^{2}$ of the two edge-states of Fig. 4 d.

that the inverted band ordering of $\mathrm{HgTe}$ is restored and the absolute value of the negative band gap is increasing (see Fig. 6).

At a $\mathrm{HgTe}$ well width of $8.2 \mathrm{~nm}$, the $s$-type band drops even below the second confined $p$-type state. This reordering of bands with well thickness is summarized in Table II and illustrated in Fig. 6.

TABLE II: Orbital character of the top most valence band and lowest conduction band in $\mathrm{CdTe}-\mathrm{HgTe}-\mathrm{CdTe}$ heterostructure depending on the well width $\mathrm{d}_{Q W}$. The critical well width $\mathrm{d}_{c}$ is the equal to $6.3 \mathrm{~nm}$.

\begin{tabular}{|c|c|c|}
\hline HgTe Well thickness & Highest Val.Band & Lowest Cond.Band \\
\hline $\mathrm{d}_{Q W}<\mathrm{d}_{C}$ & p-type & s-type \\
\hline $8.2 \mathrm{~nm}>\mathrm{d}_{Q W}>\mathrm{d}_{C}$ & s-type & p-type \\
\hline $\mathrm{d}_{Q W}>8.2 \mathrm{~nm}$ & p-type & p-type \\
\hline
\end{tabular}

\section{A. Stoichiometric and Temperature Control of Critical Width}

In the previous sections, it has been shown that the effective band gap of the $\mathrm{CdTe} / \mathrm{HgTe} / \mathrm{CdTe}$ quantum well depends on the confinement and consequently on the band gap difference of the well and barrier materials. Both, alloying and temperature are known to influence the effective band gap. The band gap of $\mathrm{Cd}_{x} \mathrm{Hg}_{1-x} \mathrm{Te}$ as a function of temperature [25] $T$ and stoichiometry $x$ is given by

$$
E_{g}=-304+\frac{0.63 T^{2}}{11+T}(1-2 x)+1858 x+54 x^{2} .
$$

A plot for the band-gap variation for the $\mathrm{Cd}_{x} \mathrm{Hg}_{1-x} \mathrm{Te}$ alloy is given in Fig. 7.

When the quantum well material of the original $\mathrm{CdTe} / \mathrm{HgTe} / \mathrm{CdTe}$ structure is substituted by $\mathrm{Cd}_{x} \mathrm{Hg}_{1-x} \mathrm{Te}$ alloy, the critical width becomes temperature and $x$ dependent. This is shown in Fig. 5] (a). Remarkably, all critical widths are equal or larger than the intrinsic critical width of $6.3 \mathrm{~nm}$. Higher concentration of CdTe in the quantum well reduces the Darwin 


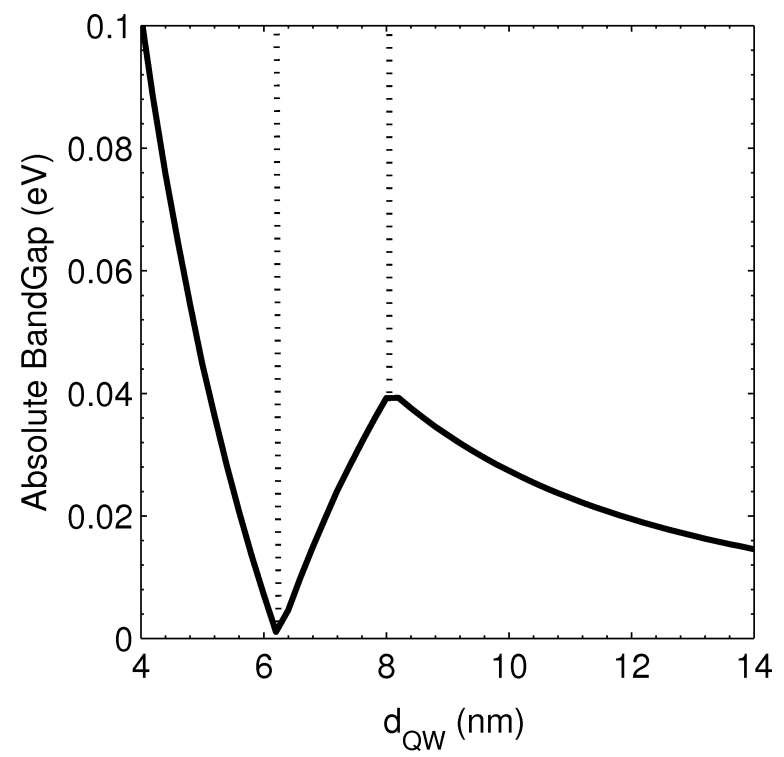

FIG. 6: Absolute value of the band gap of a $\mathrm{CdTe} / \mathrm{HgTe} / \mathrm{CdTe}$ quantum well as a function of the well width. Well widths larger than $6.3 \mathrm{~nm}$ produce inverted band structures and can be exploited for topological insulator devices.

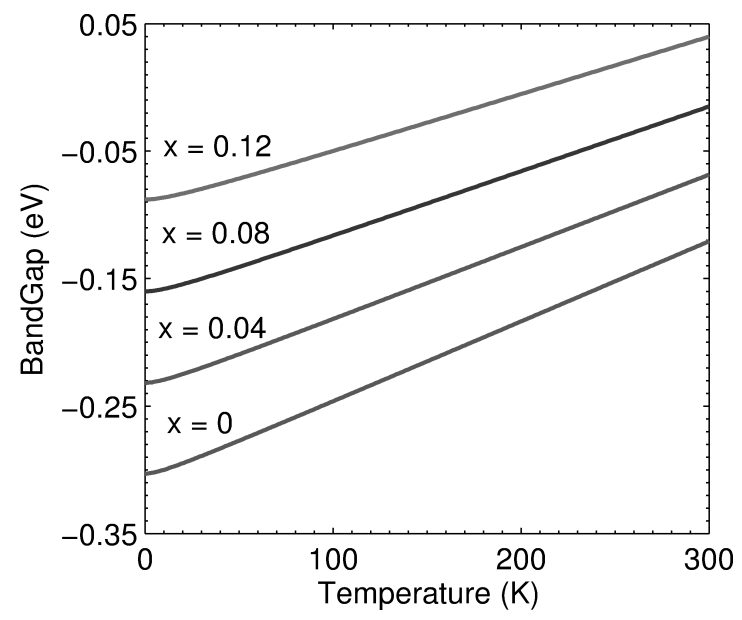

FIG. 7: Calculated band gap of bulk $\mathrm{Cd}_{x} \mathrm{Hg}_{1-x} \mathrm{Te}$ as a function of stoichiometry and temperature. At $x=0$, the bulk band gap of HgTe $(-0.303 \mathrm{eV})$ is reproduced.

contribution from HgTe. Therefore, the band inversion requires a wider $\mathrm{HgTe}$ region.

Alternatively, replacing the barrier material with $\mathrm{Cd}_{x} \mathrm{Hg}_{1-x}$ Te also allows tuning the confinement and consequently the critical width. It is shown in Fig. 7(b) that this replacement yields critical widths smaller then the intrinsic $6.3 \mathrm{~nm}$ if the temperature is allowed to attain values below $100 \mathrm{~K}$. For a Cd molar concentration of $x=0.68$ and $T=0 \mathrm{~K}$, the critical width dropped to $4.4 \mathrm{~nm}$. This is due to the enhanced Darwin contribution to the electronic properties with increased $\mathrm{Hg}$ content.
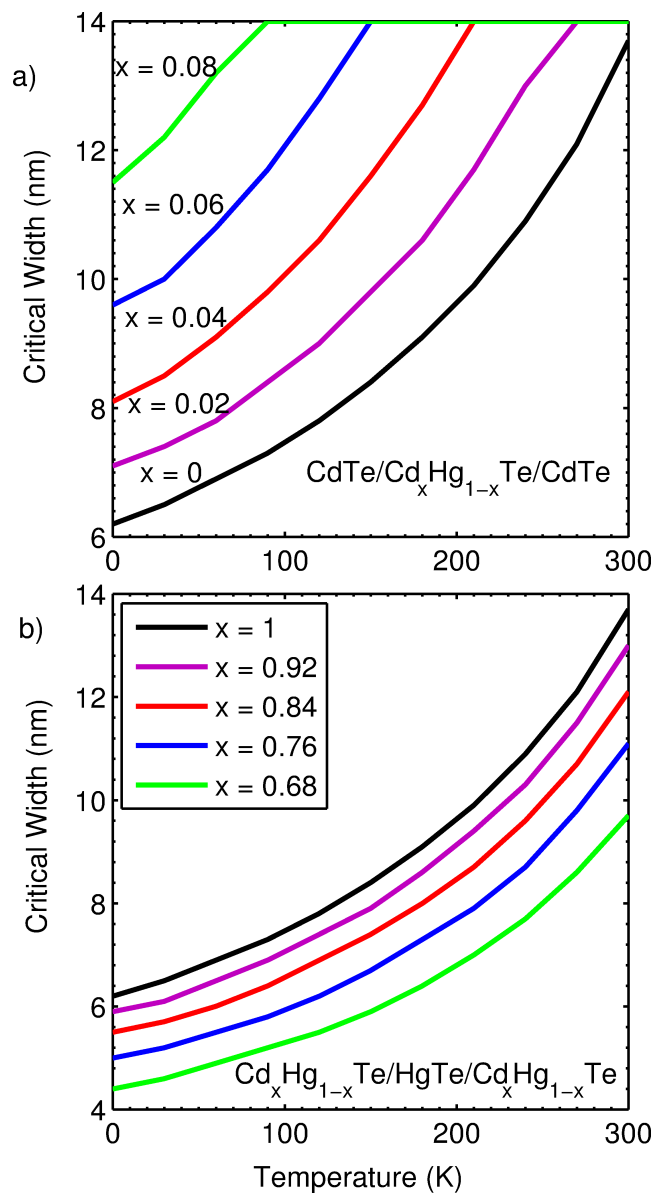

FIG. 8: Critical widths to get inverted band structures of $\mathrm{CdTe} / \mathrm{Cd}_{1-x} \mathrm{Hg}_{x} \mathrm{Te} / \mathrm{CdTe}$ quantum wells (a) and $\mathrm{Cd}_{x} \mathrm{Hg}_{1-x} \mathrm{Te} / \mathrm{HgTe} / \mathrm{Cd}_{x} \mathrm{Hg}_{1-x} \mathrm{Te}$ quantum wells (b) as a function of temperature and stoichiometry $x$. 
TABLE III: The optimal tensile stress and growth conditions for $\mathrm{CdTe} / \mathrm{HgTe} / \mathrm{CdTe}$ quantum wells to achieve the least (L), highest $(\mathrm{H})$ and intermediate (I) critical width, respectively.

\begin{tabular}{|c|c|c|c|}
\hline & \multicolumn{3}{|c|}{ Tensile uniaxial stress } \\
\hline Growth Axis & $\langle 001\rangle$ & $\langle 110\rangle$ & $\langle 111\rangle$ \\
\hline$\langle 001\rangle$ & $\mathrm{L}$ & $\mathrm{H}$ & $\mathrm{I}$ \\
\hline$\langle 110\rangle$ & $\mathrm{L}$ & $\mathrm{H}$ & $\mathrm{I}$ \\
\hline$\langle 111\rangle$ & $\mathrm{H}$ & $\mathrm{L}$ & $\mathrm{I}$ \\
\hline
\end{tabular}

\section{B. Critical widths under different growth conditions}

Apart from alloy stoichiometry, the confinement also depends on the well and barrier masses. A way to tune these effective confinement masses is by growing the quantum well in different directions. The different masses then give different effective well confinement and accordingly different critical widths. This dependence is illustrated in Fig. 9. It shows the critical width of the $\mathrm{CdTe} / \mathrm{HgTe} / \mathrm{CdTe}$ quantum well in a sequence of growth directions. The critical widths of the $\langle 111\rangle$ and $\langle 110\rangle$ growth directions are $5.52 \mathrm{~nm}$ and $5.72 \mathrm{~nm}$ respectively. Both these values are smaller than the $\langle 001\rangle$ grown quantum well critical width of $6.3 \mathrm{~nm}$.
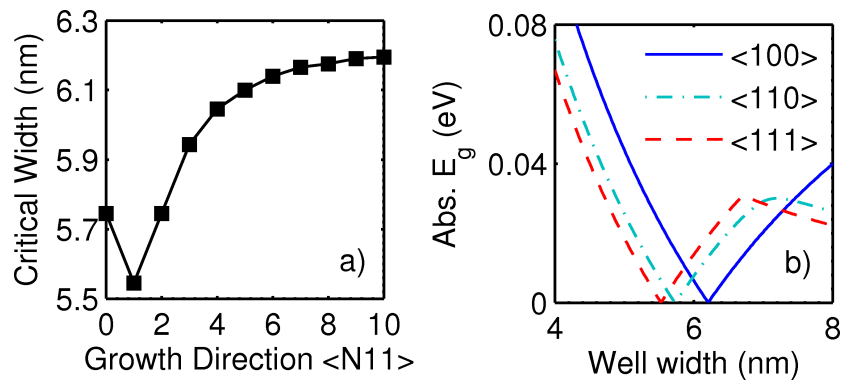

FIG. 9: Critical widths of $\mathrm{CdTe} / \mathrm{HgTe} / \mathrm{CdTe}$ heterostructures grown along $\langle N 11\rangle$ direction as a function of $N$ (a). The bandgap closing for $\langle 100\rangle,\langle 110\rangle$, and $\langle 111\rangle$ grown $\mathrm{CdTe} / \mathrm{HgTe} / \mathrm{CdTe}$ at different well widths is shown in (b). Band gap closing at different well dimensions give the corresponding critical width.

Alternatively, uniaxial stress can also tune the effective confinement masses. As representative cases, $\mathrm{CdTe} / \mathrm{HgTe} / \mathrm{CdTe}$ quantum wells were grown along $\langle 001\rangle,\langle 110\rangle$, and $\langle 111\rangle$ directions. Each quantum well was then subjected to uniaxial stress along $\langle 001\rangle$, $\langle 110\rangle$, and $\langle 111\rangle$ directions. Uniaxial stress along $\langle 001\rangle,\langle 110\rangle$, and $\langle 111\rangle$ was employed on three sets of $\mathrm{CdTe} / \mathrm{HgTe} / \mathrm{CdTe}$ quantum wells grown along $\langle 001\rangle$, $\langle 110\rangle$, and $\langle 111\rangle$. The behaviour of the critical width for each case is shown in Fig. 10. The ideal stress orientation for each growth direction is summarized in Tables II and IV.

\section{Application of an external electric field}

The application of an external electric field changes the confinement and the band properties of the well states. In particular, the Rashba (structural inversion asymmetry)
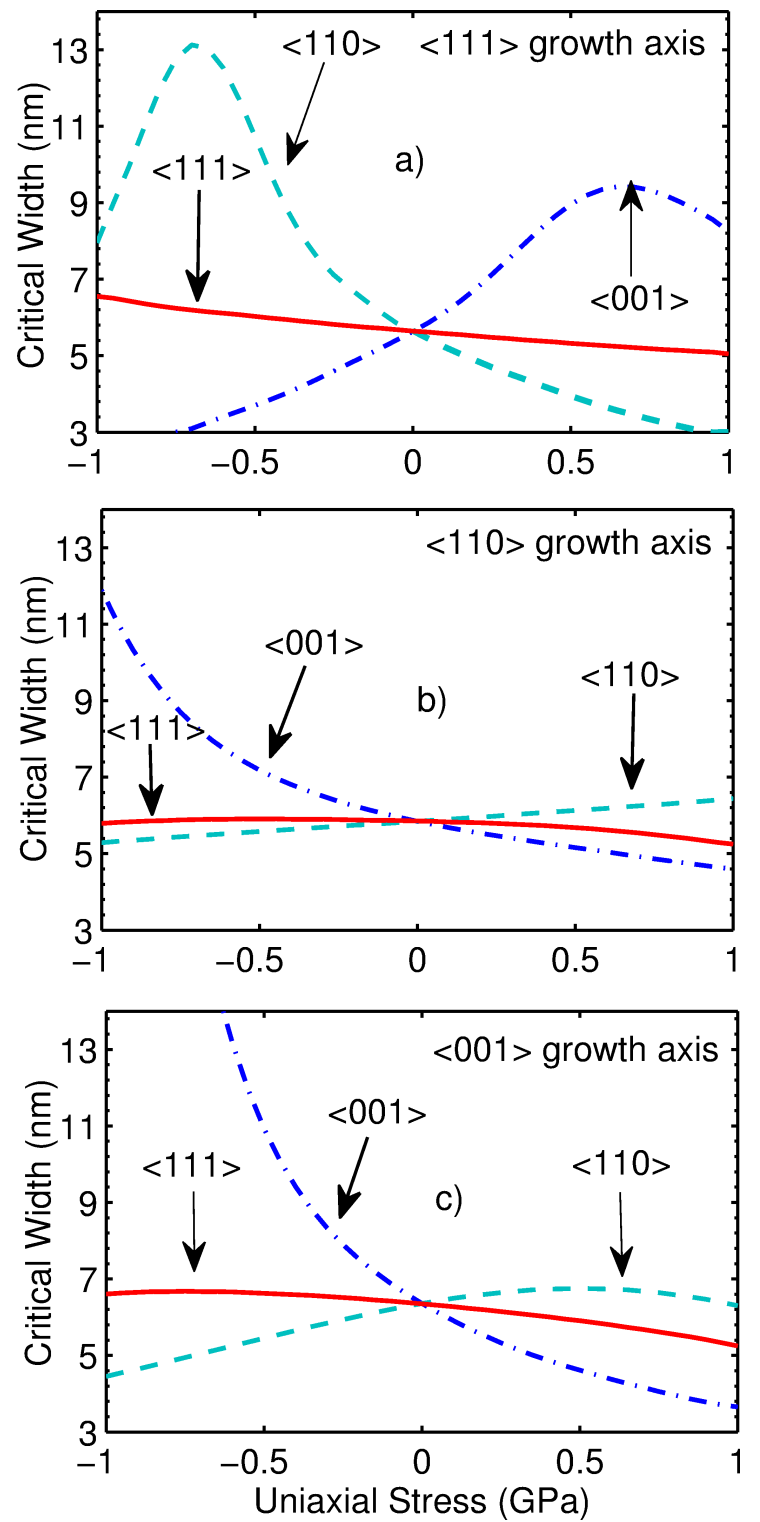

FIG. 10: Critical widths of $\mathrm{CdTe} / \mathrm{HgTe} / \mathrm{CdTe}$ heterostructures grown along $\langle 111\rangle(\mathrm{a}),\langle 110\rangle(\mathrm{b})$, and $\langle 001\rangle$ (c) direction with uniaxial stress applied along $\langle 111\rangle$ (solid), $\langle 110\rangle$ (dashed) and $\langle 001\rangle$ (dash-dotted) direction. Key observations are summarized in Table III and table IV

effect gets enhanced by electric fields in growth direction. 26] Figure 11] shows the critical width as function of the external electric field applied in the growth direction. The critical width decays with increasing field for all considered temperatures. The Rashba effect that supports the band inversion of $\mathrm{HgTe}$ gets increased by the electric field. Consequently, smaller well widths are required to invert the $\mathrm{CdTe} / \mathrm{HgTe} / \mathrm{CdTe}$ quantum well band structure when external electric fields are present.

Since external electric fields can tune the critical width, the concept of a TI-switch is obvious: A $\mathrm{CdTe} / \mathrm{HgTe} / \mathrm{CdTe}$ quantum well with a well width that is close, but below the critical width can be switched 
TABLE IV: The same list of conditions as in Table III but under compressive stress.

\begin{tabular}{|c|c|c|c|}
\hline & \multicolumn{3}{|c|}{ Compressive uniaxial stress } \\
\hline Growth Axis & $\langle 001\rangle$ & $\langle 110\rangle$ & $\langle 111\rangle$ \\
\hline$\langle 001\rangle$ & $\mathrm{H}$ & $\mathrm{L}$ & $\mathrm{I}$ \\
\hline$\langle 110\rangle$ & $\mathrm{H}$ & $\mathrm{L}$ & $\mathrm{I}$ \\
\hline$\langle 111\rangle$ & $\mathrm{L}$ & $\mathrm{H}$ & $\mathrm{I}$ \\
\hline
\end{tabular}

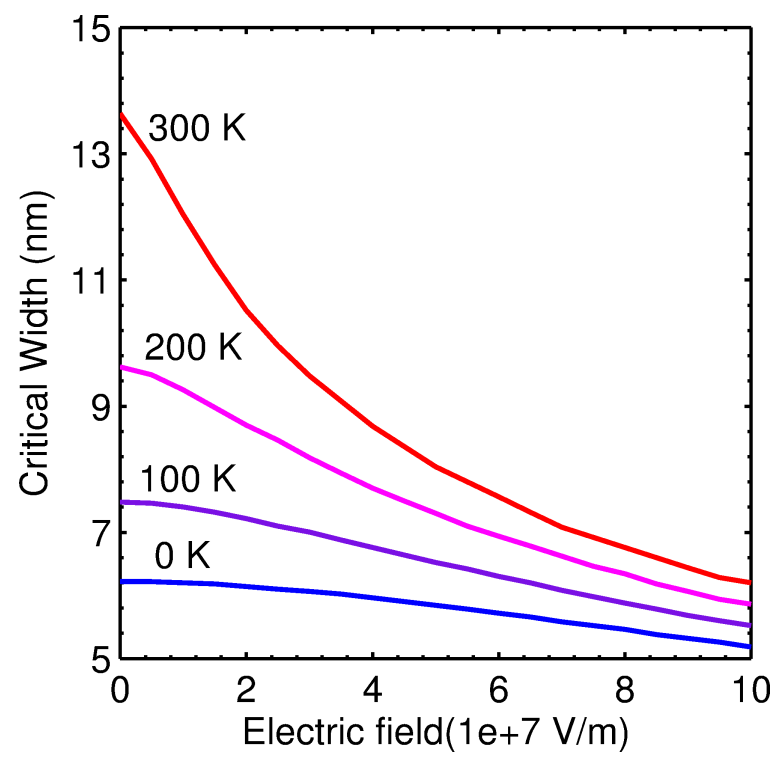

FIG. 11: Critical width for $\mathrm{CdTe} / \mathrm{HgTe} / \mathrm{CdTe}$ quantum wells with varying strength of external electric fields in growth direction.

by electric fields between normal and inverted band order. Such a switching band structure is expected to yield significant changes in the surface conductance, due to the unique transport properties of topological insulator states.

A first prototype of such a switch can be observed in Fig. 12 which shows effective band gaps of $\mathrm{CdTe} / \mathrm{HgTe} / \mathrm{CdTe}$ quantum wells for various well thicknesses under externally applied electric fields. Within the plotted range of electric field magnitude, the $\mathrm{CdTe} / \mathrm{HgTe} / \mathrm{CdTe}$ quantum well with a width of $6.0 \mathrm{~nm}$ switches between normal and inverted band ordering. It is worth mentioning that this switching behavior can be observed in $\mathrm{CdTe} / \mathrm{HgTe} / \mathrm{CdTe}$ quantum wells grown in $\langle 001\rangle$ and $\langle 111\rangle$ direction.

Such topological insulator based devices under an external electric field can be employed to act as a circuit element in a fast digital environment. When the bandgap is closed and TI properties are turned on, a high Fermi velocity for the carriers, (which is an essential attribute of TIs) on surface is able to transmit an electric signal faster than a conventional inter-connect. A seamless transition from a topological insulator to normal insulator using an external electric field as demonstrated above and shown in Fig. 12 enables it to forbid an easy passage of charge/electric signal. A normal insulator with a finite band gap will behave as an open circuit element.

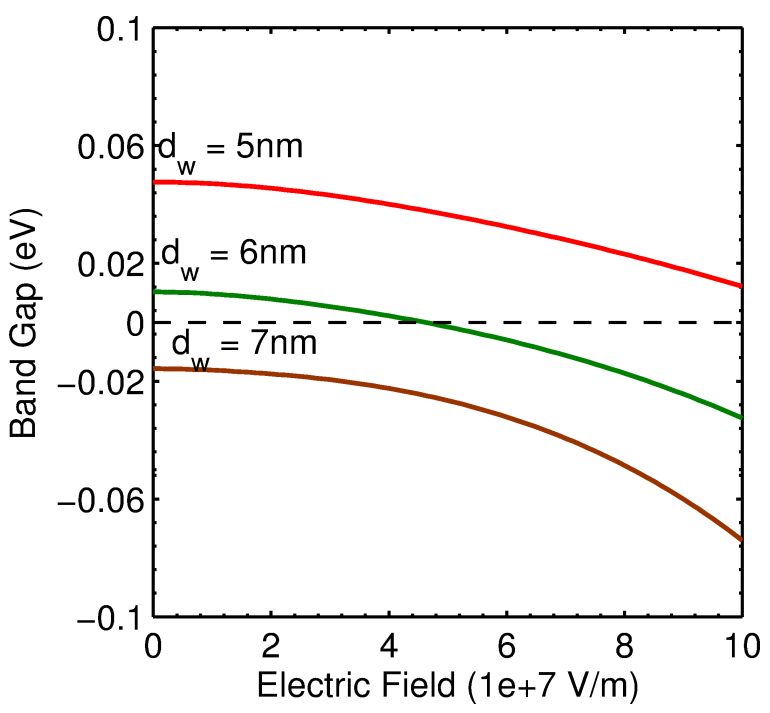

FIG. 12: Effective band gap of $\mathrm{CdTe} / \mathrm{HgTe} / \mathrm{CdTe}$ quantum wells of different well thicknesses as a function of applied electric field in growth direction. The dashed line depicts the delimiter between normal and inverted band structures.

\section{Conclusion}

The present work investigates the conditions under which band inversion can occur in a $\mathrm{CdTe} / \mathrm{HgTe} / \mathrm{CdTe}$ quantum well heterostructure. It is shown that this band inversion is essential for topological insulator properties. In agreement with experimental results, it is found that the $\mathrm{HgTe}$ quantum well has to be thicker than $6.3 \mathrm{~nm}$ to exhibit topological insulator properties. It is examined in detail how the critical width depends on various device parameters such as the growth direction, alloy stoichiometry, temperature, uniaxial stress, and external electric fields. In particular the external fields allow to switch the topological insulator properties of $\langle 001\rangle$ grown $\mathrm{CdTe} / \mathrm{HgTe} / \mathrm{CdTe}$ quantum wells. This result proposes a new class of switching devices.
[1] M. Z. Hasan and C. L. Kane, Rev. Mod. Phys. 82, 3045 (2010).

[2] L. Fu and C. L. Kane, Phys. Rev. B 76, 045302 (2007).

[3] R. Roy, Phys. Rev. B 79, 195322 (2009).
[4] C. L. Kane and E. J. Mele, Phys. Rev. Lett. 95, 146802 (2005).

[5] S. Murakami, New Journal of Physics 9, 356 (2007).

[6] H. Zhang, C. Liu, X. Qi, X. Dai, Z. Fang, and S. Zhang, 
Nature Physics 5, 438 (2009).

[7] Y. Xia, D. Qian, D. Hsieh, L. Wray, A. Pal, H. Lin, A. Bansil, D. Grauer, Y. Hor, R. Cava, et al., Nature Physics 5, 398 (2009).

[8] Y. Chen, J. Analytis, J. Chu, Z. Liu, S. Mo, X. Qi, H. Zhang, D. Lu, X. Dai, Z. Fang, et al., Science 325, 178 (2009).

[9] M. König, S. Wiedmann, C. Brüne, A. Roth, H. Buhmann, L. Molenkamp, X. Qi, and S. Zhang, Science 318, 766 (2007).

[10] B. Bernevig, T. Hughes, and S. Zhang, Science 314, 1757 (2006).

[11] A. Roth, C. Brüne, H. Buhmann, L. Molenkamp, J. Maciejko, X. Qi, and S. Zhang, Science 325, 294 (2009).

[12] M. Büttiker, Science 325, 278 (2009).

[13] N. Cade and P. Lee, Solid state communications 56, 637 (1985).

[14] I. Tsidil'kovskii, Band Structure of Semiconductors (Pergamon Press, 1982), ISBN 9780080216577.

[15] L. Molenkamp, (Private Communication).

[16] J. Maciejko, T. Hughes, and S. Zhang, Annu. Rev. Condens. Matter Phys. 2, 31 (2011).

[17] P. Sengupta, S. Lee, S. Steiger, H. Ryu, and G. Klimeck, in MRS Proceedings (Cambridge Univ Press, 2011), vol. 1370.

[18] E. G. Novik, A. Pfeuffer-Jeschke, T. Jungwirth, V. Latussek, C. R. Becker, G. Landwehr, H. Buhmann, and L. W. Molenkamp, Phys. Rev. B 72, 035321 (2005).

[19] J. Schulman and Y. Chang, Physical Review B 33, 2594 (1986).
[20] G. Wu and T. McGill, Applied Physics Letters 47, 634 (1985).

[21] J. Lu, W. Shan, H. Lu, and S. Shen, New Journal of Physics 13, 103016 (2011).

[22] C. Brüne, A. Roth, H. Buhmann, E. Hankiewicz, L. Molenkamp, J. Maciejko, X. Qi, and S. Zhang, Arxiv preprint arXiv:1107.0585 (2011).

[23] M. König, H. Buhmann, L. Molenkamp, T. Hughes, C. Liu, X. Qi, and S. Zhang, Arxiv preprint arXiv:0801.0901 (2008).

[24] J. Teo, L. Fu, and C. Kane, Physical Review B 78, 045426 (2008).

[25] S. Krishnamurthy, A. Chen, A. Sher, and M. Van Schilfgaarde, Journal of electronic materials 24, 1121 (1995).

[26] X. Zhang, A. Pfeuffer-Jeschke, K. Ortner, V. Hock, H. Buhmann, C. Becker, and G. Landwehr, Physical Review B 63, 245305 (2001).

\section{Acknowledgments}

Computational resources from nanoHUB.org and support by National Science Foundation (NSF) (Grant Nos. EEC-0228390, OCI-0749140) are acknowledged. This work was also supported by the Semiconductor Research Corporation's (SRC) Nanoelectronics Research Initiative and National Institute of Standards \& Technology through the Midwest Institute for Nanoelectronics Discovery (MIND), SRC Task 2141, and Intel Corporation. 

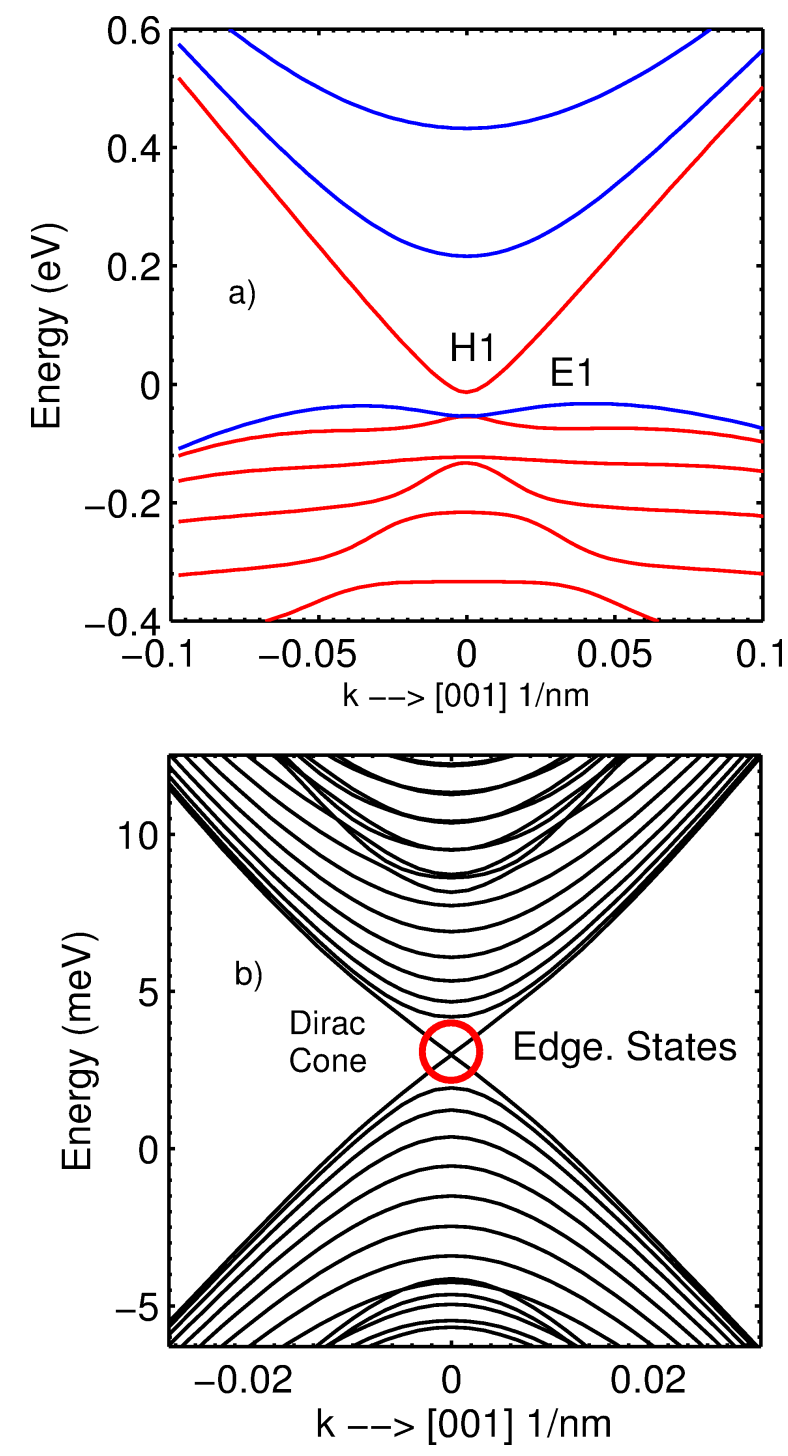